\title{
Repeatability and reproducibility of right ventricular Tei index valves derived from three echocardiographic methods for evaluation of cardiac function in dogs
}

\begin{abstract}
Objective: To evaluate repeatability and reproducibility of right ventricular Tei index (RTX) values derived from dual pulsed-wave Doppler, conventional pulsed-wave Doppler, and tissue Doppler echocardiography and to investigate relationships and repeatability among the 3 methods in healthy dogs.

Animals: 6 healthy adult Beagles.

Procedure: Echocardiography was performed on each dog on different days for 2 weeks (3 times/d) by 2 echocardiographers. Intraobserver within- and between-day and interobserver coefficients of variation (CVs) and intraclass correlation coefficients (ICCs) for RTXs derived from dual pulse-waved Doppler $\left(\mathrm{RTX}_{\mathrm{DPD}}\right)$, conventional pulsed-wave Doppler ( $\left.\mathrm{RTX}_{\mathrm{PD}}\right)$, and tissue Doppler $\left(\mathrm{RTX}_{\mathrm{TD}}\right)$ methods were determined. Degrees of agreement among RTX values derived from the 3 methods were assessed by modified Bland-Altman analysis.
\end{abstract}

Results: Least squares mean (95\% confidence interval) $\mathrm{RTX}_{\text {td }}$ was 0.50 (0.46 to 0.54$)$, which was significantly higher than that for $\operatorname{RTX}_{\mathrm{DPD}}(0.27$ [0.23 to 0.31$\left.]\right)$ and $\mathrm{RTX}_{\mathrm{PD}}(0.25$ [0.21 to 0.29]). Agreement between RTX $\mathrm{DPD}_{\mathrm{P}}$ and RTX $\mathrm{RD}$ was good (bias [mean difference], 0.04 [95\% confidence interval, -0.03 to 0.10$])$. The $\mathrm{RTX}_{\text {dpd }}$ had high within-day $(\mathrm{CV}, 6.1$; ICC, $0.77)$ and interobserver $(\mathrm{CV}, 3.5$; ICC, 0.83$)$ repeatability, but between-day repeatability was not high. The $\mathrm{RTX}_{\mathrm{td}}$ had high within-day repeatability $(\mathrm{CV}, 6.0 ; \mathrm{ICC}, 0.80)$, but between-day and interobserver repeatability were not high. Within-day, between-day, and interobserver repeatability of RTXPD were not high.

Conclusions and clinical relevance: $\mathrm{RTX}_{\mathrm{dpd}}$ measurement was a repeatable and reproducible method of cardiac evaluation in healthy dogs. The $\mathrm{RTX}_{\mathrm{TD}}$ values were significantly higher than the $\mathrm{RTX}_{\mathrm{DPD}}$ and $\mathrm{RTX}_{\mathrm{PD}}$ values; therefore, RTX values derived from different echocardiographic methods should be interpreted with caution.

Keyword: Echocardiographic methods; Cardiac function; Dogs 